\title{
An Analysis of the Femoral Drilling Angle to Avoid Tunnel Collision during Double-Bundle Anterior Cruciate Ligament and Anterolateral Ligament Reconstruction on the Knee
}

\author{
Yusuke Kawanishi, MD, $\mathrm{PhD}^{1}$ Makoto Kobayashi, MD, $\mathrm{PhD}^{1}$ Sanshiro Yasuma, MD, $\mathrm{PhD}^{1}$ (1) \\ Hiroaki Fukushima, $\mathrm{MD}^{1}$ jiro Kato, $\mathrm{MD}^{1}$ Atsunori Murase, MD, $\mathrm{PhD}^{2}$ Tetsuya Takenaga, MD, $\mathrm{PhD}^{1}$ \\ Masahito Yoshida, MD, PhD ${ }^{1}$ Gen Kuroyanagi, MD, PhD ${ }^{1}$ Yohei Kawaguchi, MD, PhD ${ }^{1}$ \\ Yuko Nagaya, MD, $\mathrm{PhD}^{1}$ Hideki Murakami ${ }^{1}$ Masahiro Nozaki, MD, PhD ${ }^{1}$
}

\footnotetext{
1 Department of Orthopedic Surgery, Nagoya City University Graduate School of Medical Sciences and Medical School, Mizuho-Cho Mizuho-Ku, Nagoya, Japan

2 Department of Orthopedic Surgery, Kasugai Joint \& Sports Orthopedic Clinic, Kasugai, Aichi, Japan
}

J Knee Surg 2023;36:483-490.
Address for correspondence Masahiro Nozaki, MD, PhD, Department of Orthopedic Surgery, Nagoya City University Graduate School of Medical Sciences, 1-Kawasumi, Mizuho-Cho, Mizuho-Ku, Nagoya 4678601, Japan (e-mail: mnozaki@med.nagoya-cu.ac.jp).

\begin{abstract}
Keywords

- anterior cruciate ligament

- anterolateral ligament reconstruction

- tunnel collision

- knee

Concomitant anterior cruciate ligament (ACL) and anterolateral ligament (ALL) reconstruction has been reported as an effective technique for providing rotational control of the knee. However, the intraoperative risk of collision with an $\mathrm{ACL}$ tunnel during the drilling for the femoral ALL tunnel has been described. The purpose of this study was to investigate the various femoral drilling procedures to avoid tunnel collisions during combined double-bundle ACL and ALL reconstruction. Nine cadaveric knees were used in this study. ACL drilling was performed through the anteromedial portal to footprints of the posterolateral bundle at $120^{\circ}$ (PL120) and $135^{\circ}$ (PL135) knee flexion and the anteromedial bundle at $120^{\circ}$ (AM120) and $135^{\circ}$ (AM135) knee flexion. ALL drilling was performed at $0^{\circ}$ (Cor0-ALL) and $30^{\circ}$ (Cor30-ALL) coronal angles using a Kirschner wire (K-wire). The distance between the ALL footprint and ACL K-wire outlets, axial angles of ALL K-wires colliding with ACL K-wires, and distances from the ALL footprint to the collision point were measured. From these values, the safe zone, defined as the range of axial angles in which no collisions or penetrations occurred, was identified by simulation of tunnels utilized for reconstruction grafts in each drilling procedure. The point-to-point distance from the ALL footprint to the K-wire outlet was significantly greater in the AM120 than the AM135 $(13.5 \pm 3.1,10.8 \pm 3.2 \mathrm{~mm} ; p=0.048)$ and in the PL135 than the PL120 $(18.3 \pm 5.5,16.1 \pm 6.5 \mathrm{~mm} ; p=0.005)$ conditions, respectively. During an ACL drilling combination of PL135/AM120, a safe zone of $>45^{\circ}$ in Cor30-ALL was identified. With a narrow safe zone during the PL135/AM120 combination only, the risk of femoral tunnel collisions in combined double-bundle $A C L$ and $A L L$ reconstruction is high. AM drilling at $120^{\circ}$ and PL drilling at $>135^{\circ}$ knee flexion, combined with ALL drilling at $30^{\circ}$ coronal angle and $>45^{\circ}$ axial angle, may reduce this risk.
\end{abstract}

received

October 26, 2020

accepted after revision

August 18, 2021

article published online

October 8, 2021 (c) 2021. Thieme. All rights reserved.

Thieme Medical Publishers, Inc.,

333 Seventh Avenue, 18th Floor,

New York, NY 10001, USA
DOI https://doi.org/

10.1055/s-0041-1736196. ISSN 1538-8506. 
The anterior cruciate ligament (ACL) is frequently injured in the athletic populations. ACL reconstruction has been considered as a treatment for controlling anteroposterior and rotational stability. ${ }^{1}$ Recently, the anterolateral ligament (ALL) has been reported as a secondary stabilizer to the ACL in several biomechanical studies. ${ }^{2-4}$ The ALL also provides rotational control of the knee during the simulated pivot shift ${ }^{2}$ and in resisting anterior tibial translation ${ }^{4}$ and internal tibial rotation. ${ }^{3}$ A cadaveric biomechanical study has suggested that ALL injury may contribute to residual internal rotation and a positive pivot shift following ACL reconstruction. ${ }^{2}$ Preoperative ALL injury was associated with worse clinical outcomes after ACL reconstruction compared with isolated ACL injury. ${ }^{5}$ Concomitant ACL and ALL reconstruction has been shown to reduce internal rotation and axial plane tibial translation compared with isolated ACL reconstruction for ACL- and ALL-deficient knees. ${ }^{6}$ Moreover, combined ACL and ALL reconstruction has been reported as an effective technique for achieving a higher rate of return to preinjury sport levels, ${ }^{7-9}$ reduction in graft failures, ${ }^{8-10}$ and better knee stability $7,8,10$ compared with isolated ACL reconstruction.

Although various techniques for ALL reconstruction have been reported, ${ }^{11-13}$ the risk of collision with an ACL tunnel during drilling for the femoral ALL tunnel has been described. ${ }^{11,14}$ Numerous studies have evaluated the risk of femoral tunnel collision between the ACL and the fibular collateral ligament $(\mathrm{FCL})^{15-17}$ for multiligament reconstruction; however, there have been few reports on femoral tunnel collisions in combined ACL and ALL reconstruction. ${ }^{14,18}$ Furthermore, these studies on femoral tunnel collisions have evaluated single-bundle ACL and ALL reconstruction procedures $^{14,18}$ without assessing combined double-bundle ACL and ALL reconstruction. Although the superiority of double-bundle over single-bundle $\mathrm{ACL}$ reconstruction remains controversial, some reports have stated that double-bundle ACL reconstruction is superior in decreasing the risk of graft failures ${ }^{19}$ and in providing better knee stability. ${ }^{20,21}$ Therefore, it is important to establish procedures that aid in avoiding femoral tunnel collisions during combined double-bundle ACL and ALL reconstruction.

The purpose of this study was to investigate the various drilling procedures to avoid collisions between femoral ACL and ALL tunnels during combined double-bundle ACL and ALL reconstruction. We hypothesized that very specific ALL drilling angles would be necessary to ensure an optimal drilling procedure, in order to avoid collisions during combined reconstructions.

\section{Materials and Methods}

This study was approved by the institutional review board of the affiliated institutions.

\section{Subjects}

Nine cadaveric knees (four male and five female cadavers from a 2019 gross anatomy course) embalmed with formaldehyde were used for this study. All had intact cruciate ligaments, and none presented with macroscopic evidence of fractures, deformities, or joint contractures. The mean age of the subjects at the time of death was $81.4 \pm 14.1$ years (range: 67-105 years). Height and weight measurements were not available. The patella, patellar tendon, skin, subcutaneous tissue above the patella and patellar tendon, infrapatellar fat pad, and ACL were removed to expose the femoral footprint of the ACL. The footprint of the posterolateral (PL) and anteromedial (AM) bundles of the ACL was identified by referring to the native ACL footprint and a previous report on the ACL femoral footprint. ${ }^{22}$

\section{ACL Drilling}

A 2.4-mm diameter Kirschner wire (K-wire) was inserted through the anteromedial portal, which was situated in the medial position as much as possible without injuring the articular cartilages and menisci (-Fig. 1A). Previous studies have described the potential risks associated with ACL femoral drilling through the anteromedial portal (e.g., injury to the common peroneal nerve, ${ }^{23,24}$ blowout of the posterior femoral cortex, ${ }^{23,25,26}$ and short femoral tunnels ${ }^{25}$ ) and have recommended that the knee be flexed $>110^{\circ}$ to decrease the risk of these complications. ${ }^{23-26}$ Based on these reports, we evaluated the knees that were flexed $120^{\circ}$ and $135^{\circ}$ in this study. The K-wire was then inserted through the footprint of the PL bundle of the ACL with the knee flexed to $120^{\circ}$ (PL120). The next K-wire was also inserted through the footprint of the PL bundle of the ACL with the knee flexed to $135^{\circ}$ (PL135). The remaining two K-wires were both inserted through the footprint of the AM bundle of the ACL: one with the knee flexed to $120^{\circ}$ (AM120) and the other with the knee flexed to $135^{\circ}$ (AM135). A total of four K-wires were inserted through the ACL footprint, and the flexion angles of the knees were measured using a goniometer. The K-wires inserted in the knees flexed to $120^{\circ}$ were pulled laterally out of the intraarticular space, so that when the knees were flexed to $135^{\circ}$, the subsequent $\mathrm{K}$-wires could be inserted through the same points. After insertion of the four wires, the knee joint and the distal third of the femur were dissected free from all soft tissues (-Fig. 1B), and the femur was placed onto a wood block.

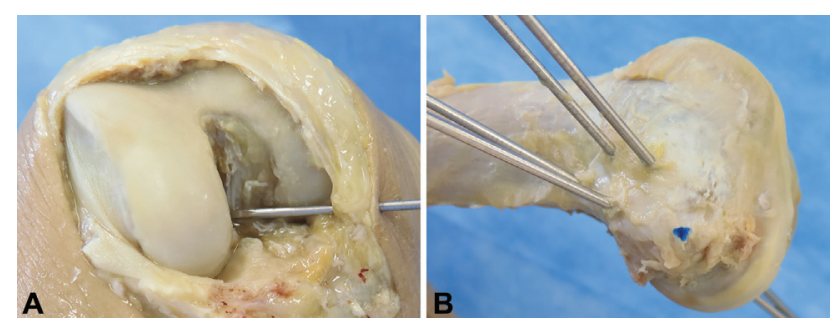

Fig. 1 Drilling procedure for the anterior cruciate ligament $(A C L)$. (A) For the anterior ACL drilling, a 2.4-mm-diameter Kirschner wire (Kwire) is inserted through the anteromedial portal, which is situated as much as possible in the medial and inferior position without injuries to the articular cartilages and menisci. (B) After insertion of the four Kwires for $A C L$ drillings, the knee joint and the distal third of the femur are dissected free from all soft tissues. The blue dot indicates the lateral epicondyle. 

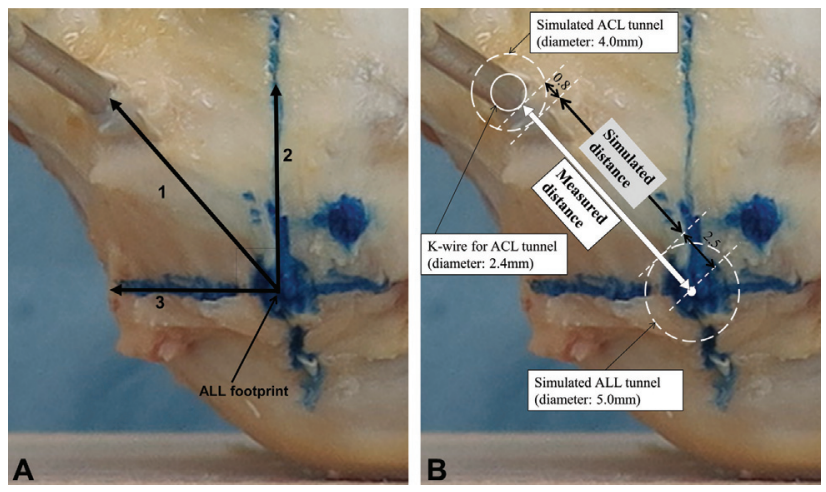

Fig. 2 Distance from the anterolateral ligament (ALL) footprint to the Kirschner wire (K-wire) outlet. Anterior-posterior and proximal-distal axes are defined as the lines perpendicular and parallel to the long axis of the femur, respectively. (A) Distance from the ALL footprint to the outlet point of the anterior cruciate ligament $(\mathrm{ACL})$ The K-wire was measured on the lateral surface of the femur: 1 ) directly (point-topoint), 2) along the anterior-posterior axis (anterior direction), and 3) along the proximal-distal axis (proximal direction). (B) The simulated distance (point-to-point) is evaluated for the collision between $\mathrm{ACL}$ and ALL tunnels in the simulation of 4.0 - and $5.0-\mathrm{mm}$ diameter $\mathrm{ACL}$ and ALL drilling, respectively, using the following formula: Simulated distance $=M_{\text {easured distance }}-3.3$.

Distance from the ALL Footprint to the K-wire Outlet The anterior-posterior and proximal-distal axes were defined as the lines perpendicular and parallel to the long axis of the femur, respectively. ${ }^{17}$ The center of the ALL footprint was identified as the point $4.0 \mathrm{~mm}$ proximal and $6.0 \mathrm{~mm}$ posterior from the lateral epicondyles, based on previous anatomic and morphologic analyses (average ALL attachment: $2.7 \mathrm{~mm}$ proximal and $2.8 \mathrm{~mm}$ posterior to the $\mathrm{FCL},{ }^{27}$ average $\mathrm{FCL}$ attachment: $1.4 \mathrm{~mm}$ proximal and $3.1 \mathrm{~mm}$ posterior to the lateral epicondyle). ${ }^{28}$ Using a ruler, we measured the distance (in $\mathrm{mm}$ ) from the center of the ALL footprint to the K-wire outlet on the lateral surface of the femur in each knee: (1) directly (point-to-point); (2) along the anterior-posterior axis (anterior direction); and (3) along the proximal-distal axis (proximal direction) ( - Fig. 2A). Considering that the tunnels utilized for reconstruction grafts require K-wires with a diameter greater than $2.4 \mathrm{~mm}$, the simulated distance $(\mathrm{mm})$ was evaluated for the collision between ACL and ALL tunnels in the simulation of 4.0- and 5.0-mm diameter ACL and ALL drilling, respectively, using the measured distance $(\mathrm{mm})$ as a reference parameter. The simulated distance was obtained using the following formula ( - Fig. 2B):

$$
S_{\text {imulated distance }}=M_{\text {easured distance }}-3.3 \text {. }
$$

$S_{\text {imulated distance }}<0$ signified collision between ACL and ALL tunnels on the lateral surface of the femur in the simulated reconstruction.

\section{ALL Drilling}

ALL femoral drilling was performed in both the coronal and axial planes. The $0^{\circ}$ coronal line was defined as the line parallel to the distal condylar line of the femur ( - Fig. 3A). The $0^{\circ}$ axial line was defined as the line parallel to the posterior condylar line of the femur ( - Fig. 3B). A 2.4-mm diameter Kwire was inserted through the ALL footprint at $0^{\circ}$ (Cor0-ALL) and $30^{\circ}$ coronal angles (Cor30-ALL) ( $\mathbf{- F i g . ~ 3 A )}$. The ALL Kwire was made to collide with each inserted ACL K-wire by adjusting the axial angles in the Cor0-ALL and Cor30-ALL orientations. The axial angles $\left({ }^{\circ}\right)$ of ALL K-wires during collisions and the distances $(D, \mathrm{~mm})$ from the ALL footprint to the collision point were measured in each knee using a protractor and another K-wire of the same length, respectively ( - Fig. 3B, C). Wood blocks configured into a cube or quadrangular prism to maintain the $0^{\circ}$ and $30^{\circ}$ coronal angles, respectively ( - Fig. 4A. B) were used to standardize ALL drilling. The wood blocks enabled us to maintain the correct orientations during drilling and improved the accuracy of our axial angle measurement. ${ }^{15}$

\section{Simulation of Collision Angle and Safe Zone in ALL Drilling}

Considering that the tunnels utilized for reconstruction grafts require K-wires with a diameter greater than
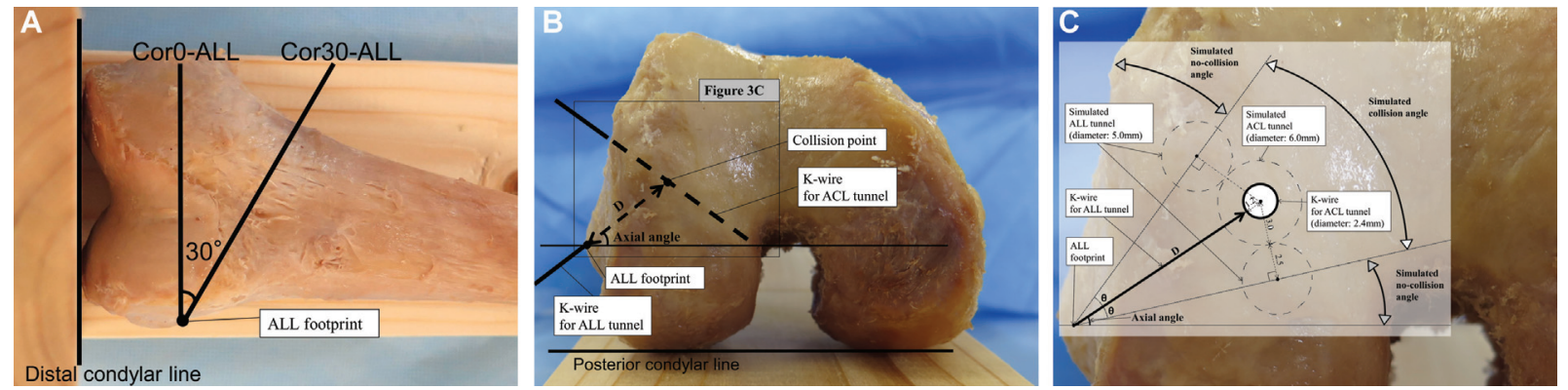

Fig. 3 Drilling procedure for the anterolateral ligament (ALL) and simulation of no-collision angles. ALL drilling is performed in both the coronal and axial planes. (A) The neutral $\left(0^{\circ}\right)$ coronal line is defined as the line parallel to the distal condylar line, and the Kirschner wire (K-wire) is inserted through the $\mathrm{ALL}$ footprint at $0^{\circ}$ (Cor0-ALL) and $30^{\circ}$ coronal angles (Cor30-ALL). (B) The neutral $\left(0^{\circ}\right)$ axial line is defined as the line parallel to the posterior condylar line, and the axial angles $\left(^{\circ}\right)$ of ALL K-wires during collisions and the distances $(D, \mathrm{~mm})$ from the ALL footprint to the collision point are measured in the Cor0-ALL and Cor30-ALL orientations. (C) This is the schema, which is an expanded form of the square area in $\mathbf{- F i g} \mathbf{3 B}$ for the range of the simulated collision angles and no-collision angles in the simulation of 6.0-mm-diameter anterior cruciate ligament (ACL) overdrilling and 5.0-mm diameter ALL drilling. The range of the simulated collision angles was obtained using the following formula: $\left(A_{x i a l}\right.$ angle $\theta)<S_{\text {imulated collision angle }}<\left(A_{\text {xial angle }}+\theta\right)$, $\sin \theta=(3.0+2.5) /(D+1.2)$. The range outside the simulated collision angles is defined as the range of the simulated no-collision angles. 

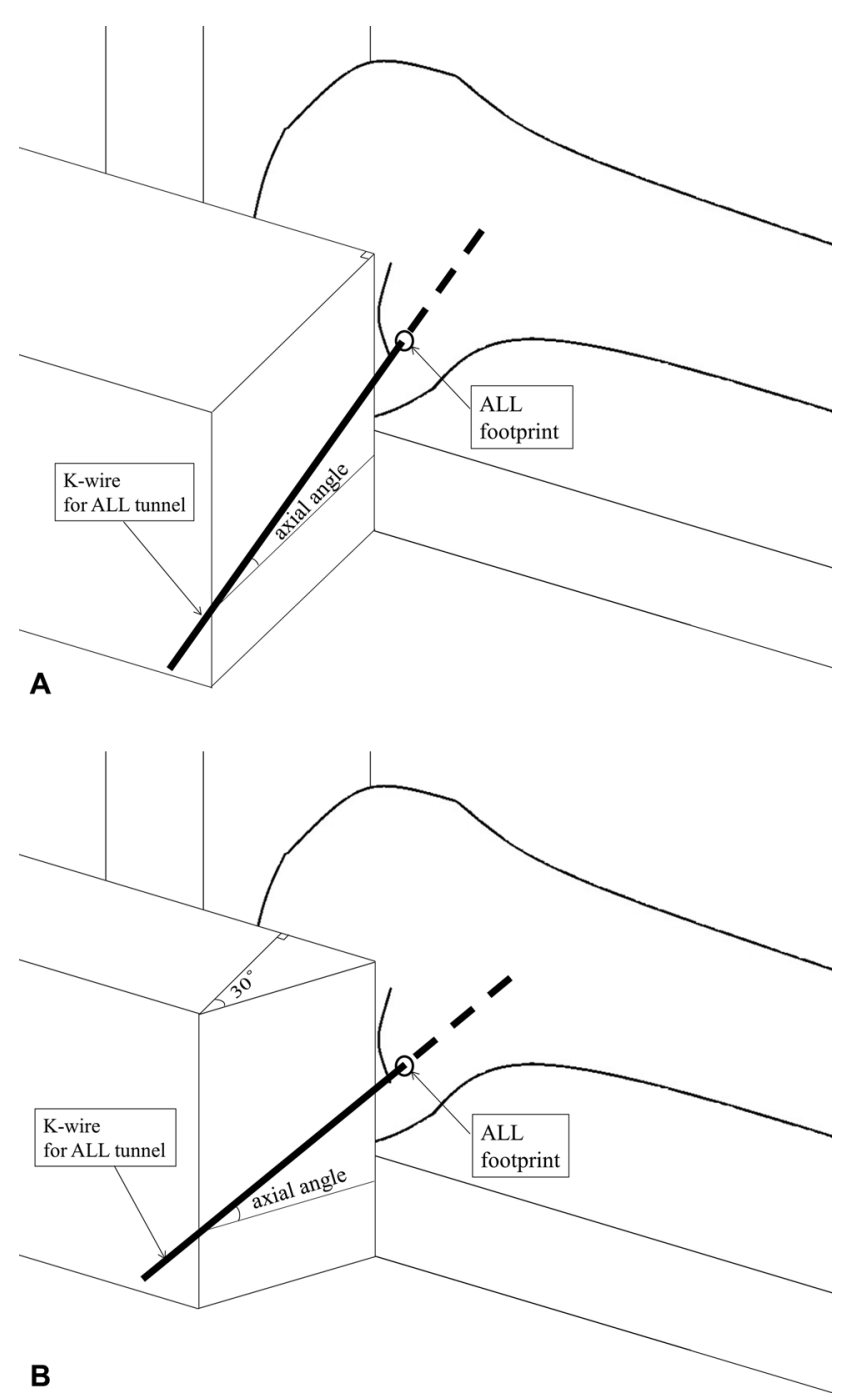

Fig. 4 Schematic representations of the wood model and the lateral face of a left knee. The two wood blocks are configured into a cube (A) or quadrangular prism (B) to maintain the required coronal angles at $0^{\circ}(\mathrm{A})$ and $30^{\circ}(\mathrm{B})$ during anterolateral ligament (ALL) drilling to improve the accuracy of our axial angle measurements.

$2.4 \mathrm{~mm}$, the range of the simulated collision angles $\left({ }^{\circ}\right)$ was evaluated in the simulation of $6.0-\mathrm{mm}$ diameter ACL overdrilling and 5.0-mm diameter ALL drilling, using the measured axial angles and $D$ during collisions in this study. The range of the simulated collision angles was obtained using the following formula ( - Fig. $3 \mathbf{C}$ ):

$\left(A_{\text {xial angle }}-\theta\right)<S_{\text {imulated collision angle }}<\left(A_{\text {xial angle }}+\theta\right)$, sin $\theta=(3.0+2.5) /(D+1.2)$.

The range outside the simulated collision angles was defined as the range of simulated no-collision angles. The common range of simulated no-collision angles in all nine knees was identified for each drilling condition: AM120 or 135, PL120 or 135, and Cor0-ALL or Cor30-ALL. Additionally, the ranges of axial angles of ALL K-wires penetrating the trochlea, intercondylar, or popliteal areas were measured, and we evaluated a safe zone, defined as the simulated nocollision angles in which the K-wires did not penetrate the trochlea, intercondylar, or popliteal areas in any of the nine knees under each drilling condition.

\section{Statistical Analysis}

Paired $t$-tests were performed to compare the distance from the ALL footprint to the K-wire outlets between AM120 and AM135 and between PL120 and PL135. In Cor0-ALL and Cor30-ALL, paired $t$-tests were performed to compare the distance from the ALL footprint to the collision point between AM120 and AM135 as well as between PL120 and PL135. A $p$-value of $<0.05$ was considered statistically significant. All statistical analyses were performed using EZR (Saitama Medical Center, Jichi Medical University, Saitama, Japan), ${ }^{29}$ which is a graphical user interface for $\mathrm{R}$ (version 2.13.0; The R Foundation for Statistical Computing, Vienna, Austria). More precisely, it is a modified version of $\mathrm{R}$ commander (version 1.6-3) designed to add statistical functions frequently used in biostatistics.

\section{Results}

\section{Safe Zone}

The axial angles of ALL K-wires during collisions and the distances from the ALL footprints to collision points are shown in -Tables $\mathbf{1}$ and $\mathbf{2}$, respectively. Based on these data, the common range of simulated no-collision angles was identified for the following combinations of drilling conditions in both Cor0-ALL and Cor30-ALL: PL120/AM120, PL120/ AM135, PL135/AM120, and PL135/AM135 (-Table 3). In Cor0-ALL, axial angles greater than $48.7^{\circ} \pm 5.8^{\circ}$ ( $\min / \max$ : $39^{\circ} / 54^{\circ}$ ) penetrated the trochlea, whereas in Cor30-ALL, no axial angles penetrated the trochlea. In Cor0-ALL, axial angles smaller than $1.4^{\circ} \pm 4.8^{\circ}\left(\min / \max :-5^{\circ} / 8^{\circ}\right)$ penetrated the intercondylar or popliteal areas, whereas in Cor30-ALL, axial angles smaller than $12.2^{\circ} \pm 4.6^{\circ}$ ( $\min / \max$ : $3^{\circ} / 18^{\circ}$ ) penetrated the intercondylar or popliteal areas. According to these findings, the axial angle ranges over which the K-wires did not penetrate the trochlea and intercondylar or popliteal area were $8^{\circ}-39^{\circ}$ in Cor0-ALL and $>18^{\circ}$ in Cor30-ALL. By applying these axial angle ranges to each simulated no-collision angle, we identified a safe zone of $>45^{\circ}$ in only Cor30-ALL during an ACL drilling combination of PL135/AM120 (- Table 3). No safe zone was identified with the other drilling combinations.

\section{Distance from the ALL Footprint to the K-wire Outlet}

The point-to-point distances from the ALL footprints to the Kwire outlets were $10.8 \pm 3.2,13.5 \pm 3.1,18.3 \pm 5.5$, and $16.1 \pm 6.5 \mathrm{~mm}$ in the PL120, PL135, AM120, and AM135 conditions, respectively. The distances between the ALL footprints and the K-wire outlets in the anterior direction were $9.2 \pm 3.1,11.4 \pm 4.2,9.9 \pm 7.0$, and $13.7 \pm 6.0 \mathrm{~mm}$ in the PL120, PL135, AM120, and AM135 conditions, respectively. Finally, the distances between the ALL footprints and the Kwire outlets in the proximal direction were $3.2 \pm 5.0$, $-4.8 \pm 4.9, \quad 14.6 \pm 3.5$, and $7.7 \pm 4.7 \mathrm{~mm}$ in the PL120, PL135, AM120, and AM135 conditions, respectively (a negative distance indicated the distal direction). The point-to- 
Table 1 Axial angles $\left(^{\circ}\right.$ ) of ALL drilling during collisions between the ALL and ACL K-wires

\begin{tabular}{|l|l|l|l|l|l|l|l|l|}
\hline \multirow{2}{*}{ Subjects } & \multicolumn{9}{l}{ Cor0-ALL } & \multicolumn{1}{l|}{ Cor30-ALL } \\
\cline { 2 - 9 } & PL120 & PL135 & AM120 & AM135 & PL120 & PL135 & AM120 & AM135 \\
\hline a & 32 & - & 3 & 8 & 55 & - & 15 & 42 \\
\hline b & 2 & 42 & 5 & 16 & 27 & - & 30 & 52 \\
\hline c & -8 & 37 & -5 & 5 & 16 & - & 8 & 18 \\
\hline d & 10 & - & -1 & -2 & 46 & - & 17 & 32 \\
\hline e & 25 & - & 13 & 37 & 64 & - & 23 & 65 \\
\hline f & - & - & 6 & 10 & - & - & 32 & 60 \\
\hline g & 47 & - & -1 & 3 & 69 & - & 8 & 40 \\
\hline h & - & - & -7 & 2 & - & - & 6 & 35 \\
\hline i & - & - & -6 & 5 & - & - & 6 & 56 \\
\hline
\end{tabular}

Abbreviations: ACL, anterior cruciate ligament; ALL, anterolateral ligament; K-wire, Kirschner wire; PL120, ACL drilling through the footprint of the posterolateral bundle in the knee flexed at $120^{\circ}$; PL135, ACL drilling through the footprint of the posterolateral bundle in the knee flexed at $135^{\circ}$; AM120, ACL drilling through the footprint of the anteromedial bundle in the knee flexed at $120^{\circ}$; AM135, ACL drilling through the footprint of the anteromedial bundle in the knee flexed at $135^{\circ}$; Cor0-ALL, ALL drilling at $0^{\circ}$ coronal angle; Cor30-ALL, ALL drilling at $30^{\circ}$ coronal angle.

Note: Raw data are presented, and all data are expressed in degrees. The symbol "-" indicates no observed collisions.

Table 2 Distances from the ALL footprint to the collision point

\begin{tabular}{|l|l|l|l|l|l|l|l|l|}
\hline \multirow{2}{*}{ Subjects } & \multicolumn{9}{l}{ Cor0-ALL } & \multicolumn{1}{l|}{ Cor30-ALL } \\
\cline { 2 - 9 } & PL120 & PL135 & AM120 & AM135 & PL120 & PL135 & AM120 & AM135 \\
\hline a & 7 & - & 28 & 25 & 5 & - & 16 & 15 \\
\hline b & 17 & 17 & 27 & 23 & 20 & - & 23 & 19 \\
\hline c & 20 & 13 & 32 & 26 & 10 & - & 23 & 20 \\
\hline d & 15 & - & 34 & 37 & 11 & - & 25 & 22 \\
\hline e & 11 & - & 17 & 12 & 9 & - & 15 & 10 \\
\hline f & - & - & 24 & 22 & - & - & 24 & 15 \\
\hline g & 10 & - & 23 & 18 & 10 & - & 10 & 7 \\
\hline h & - & - & 22 & 15 & - & - & 14 & 10 \\
\hline i & - & - & 20 & 12 & - & - & 11 & 11 \\
\hline
\end{tabular}

Abbreviations: ACL, anterior cruciate ligament; ALL, anterolateral ligament; K-wire, Kirschner wire; AM120, ACL drilling through the footprint of the anteromedial bundle in the knee flexed at $120^{\circ}$; AM135, ACL drilling through the footprint of the anteromedial bundle in the knee flexed at $135^{\circ}$; Cor0-ALL, ALL drilling at $0^{\circ}$ coronal angle; Cor30-ALL, ALL drilling at $30^{\circ}$ coronal angle; PL120, ACL drilling through the footprint of the posterolateral bundle in the knee flexed at $120^{\circ}$; PL135, ACL drilling through the footprint of the posterolateral bundle in the knee flexed at $135^{\circ}$.

Note: Raw data are presented, and all data are expressed in $\mathrm{mm}$. The symbol "-" indicates no observed collisions.

point distances were significantly longer in the PL135 condition than in the PL120 condition $(p=0.048)$. Furthermore, the point-to-point distances were significantly longer in the AM120 condition than in the AM135 condition $(p=0.005)$. In all ACL drilling conditions, the simulated distances were $>0$ $\mathrm{mm}$ in all knees. The PL135 outlet was situated in a more anterior $(p<0.001)$ and distal $(p=0.017)$ location than the PL120 outlet. The AM120 outlet was situated in a more posterior $(p<0.001)$ and proximal $(p<0.001)$ location than

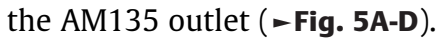

Distance from the ALL Footprint to the Collision Point The distance from the ALL footprint to the collision point was significantly longer in the AM120 condition than in the AM135 condition during Cor0-ALL (25.2 \pm 5.5 ,
$21.1 \pm 8.0 \mathrm{~mm} ; \quad p=0.005)$ and Cor30-ALL $(17.8 \pm 5.7$, $14.3 \pm 5.2 \mathrm{~mm} ; p<0.001)$. Statistical analysis could not be performed in the PL120 and PL135 conditions, as no collision was observed in several knees. No collisions were observed when the PL drilling outlets were distal to the ALL footprint (-Fig. 5A-D), and in the PL135 at any axial angles, during Cor30-ALL.

\section{Discussion}

The most important finding of the present study was that the drilling procedure that reduces the risk of femoral tunnel collisions between ACL and ALL tunnels in combined doublebundle ACL and ALL reconstruction is AM drilling at $120^{\circ}$ knee flexion and PL drilling at $135^{\circ}$ knee flexion in 
Table 3 The common range of simulated no-collision angles and safe zones for different combinations of the ACL and ALL drilling angles

\begin{tabular}{|c|c|c|c|c|}
\hline & \multicolumn{2}{|c|}{ Simulated no-collision angle $\left({ }^{\circ}\right)$} & \multicolumn{2}{|c|}{ Safe zone $\left({ }^{\circ}\right)$} \\
\hline & Cor0-ALL & Cor30-ALL & Cor0-ALL & Cor30-ALL \\
\hline PL120/AM120 & $>76$ & - & - & - \\
\hline PL120/AM135 & $>76$ & - & - & - \\
\hline PL135/AM120 & $>76$ & $>45$ & - & $>45$ \\
\hline PL135/AM135 & $>62$ & - & - & - \\
\hline
\end{tabular}

Abbreviations: ACL, anterior cruciate ligament; ALL, anterolateral ligament; AM120, ACL drilling through the footprint of the anteromedial bundle in the knee flexed at $120^{\circ}$; AM135, ACL drilling through the footprint of the anteromedial bundle in the knee flexed at $135^{\circ}$; Cor0-ALL, ALL drilling at $0^{\circ}$ coronal angle; Cor30-ALL, ALL drilling at $30^{\circ}$ coronal angle; PL120, ACL drilling through the footprint of the posterolateral bundle in the knee flexed at $120^{\circ}$; PL135, ACL drilling through the footprint of the posterolateral bundle in the knee flexed at $135^{\circ}$.

Note: All data are expressed in degrees. The symbol "-" indicates drilling combinations in which simulated no-collision angles or safe zones were not identified.
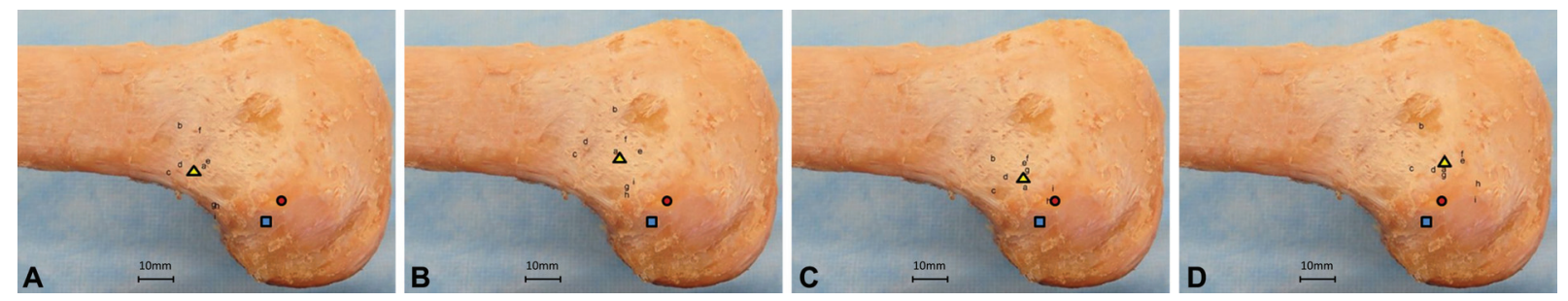

Fig. 5 Locations of Kirschner wire (K-wire) outlet points. Locations of K-wire outlet points during $(A)$ anterior cruciate ligament (ACL) drilling through the footprint of the anteromedial bundle in the knee flexed at $120^{\circ}$ (AM120), (B) ACL drilling through the footprint of the anteromedial bundle in the knee flexed at $135^{\circ}$ (AM135), (C) ACL drilling through the footprint of the posterolateral bundle in the knee flexed at 120 (PL120), and (D) ACL drilling through the footprint of the posterolateral bundle in the knee flexed at $135^{\circ}(\mathrm{PL} 135)$. The letters a-i represent outlet points for each knee. The yellow triangle indicates the average location of the nine knees; the red circle indicates the lateral epicondyle; and the blue square indicates the ALL footprint.

combination with ALL drilling at $30^{\circ}$ coronal angle and $>45^{\circ}$ axial angle. This recommendation is supported by the following results: (1) the point-to-point distance between the ALL footprint and the K-wire outlet was greater in the AM120 and PL135 conditions than in the AM135 and PL120 conditions; (2) no collision was observed in the PL135 condition in the Cor30-ALL orientation; and (3) with the PL135/AM120 combination, the safe zone was $>45^{\circ}$ in Cor30-ALL. The present study and another report ${ }^{13}$ have shown that the greater the angle of knee flexion, the more distal the location of the K-wire outlet. Furthermore, in the present study, there were no collisions when the PL drilling outlets were distal to the ALL footprint. Therefore, to place the outlets more distally from the ALL footprint, PL drilling with the knee flexed at a minimum of $135^{\circ}$ (or greater, if possible) may be better. The optimization of this procedure is clinically relevant, as it may aid surgeons by reducing concerns about tunnel collision during femoral tunnel drilling; consequently, the operation of combined double-bundle ACL and ALL reconstruction can be safely and rapidly performed. However, with the narrow safe zone $\left(>45^{\circ}\right)$ during the limited ACL drilling procedures (only PL135/AM120 combination), the results of the present study also indicate a high risk of femoral tunnel collisions in combined double-bundle ACL and ALL reconstruction, which is in support of our initial hypothesis.
There are few reports on femoral tunnel collision during combined single-bundle ACL and ALL reconstruction using computed tomography (CT). ${ }^{14,18,30}$ Jette et $\mathrm{al}^{18}$ determined that the ALL femoral tunnel should be drilled at an angle of $30^{\circ}$ anterior in the axial plane and $30^{\circ}$ proximal in the coronal plane during single-bundle ACL drilling with the knee flexed at $120^{\circ}$. Smeets et al ${ }^{14}$ observed a high risk of tunnel convergence when performing combined singlebundle ACL and ALL reconstructions, and they reported that the occurrence of tunnel conflicts can be reduced by aiming the ALL tunnel in a more proximal and anterior direction, which supports the results of the present study. The previous computed tomography studies were performed using only two types of axial angles during ALL drillings; as a result, the actual axial angles that caused collisions in each knee were not evaluated. In this study, the axial angles of ALL K-wires during actual collisions were measured, and a safe zone of ALL drilling was evaluated based on these angles.

One option to avoid collisions is to use the same combined femoral tunnel, as described in combined reconstructions of ACL and posterolateral corner, ${ }^{31}$ and posterior cruciate ligament and medial collateral ligament. ${ }^{32}$ Sonnery-Cottet et $\mathrm{al}^{33}$ described a technique of combined single-bundle $A C L$ and ALL reconstruction using the same femoral tunnel for the ACL and the ALL. A disadvantage of this technique is that it is not possible to perform double-bundle ACL 
reconstructions. Moreover, Mediavilla et $\mathrm{al}^{34}$ described a technique of combined double-bundle ACL and ALL reconstruction, using the same femoral tunnel for the PL bundle of the ACL and the ALL. As a disadvantage, the shared graft with one of the ACL bundles and ALL is a one-strand tendon graft because the graft needs to be long; thus, the diameter of the one of the ACL graft is small unless additional grafts are used. Furthermore, these techniques need to be used in an outsidein manner during femoral drilling while placing the guide to an exact position of the femoral ALL and ACL footprint, which is complicated and technical.

Some studies have investigated the relationships between the location of femoral tunnel outlets and the lateral anatomic structures (e.g., the posterior femoral cortex, attachment of the gastrocnemius, FCL, popliteus tendon, and capsule) during ACL femoral drilling through the anteromedial portal. ${ }^{17,26}$ These studies concluded that it was more desirable to drill with the knee flexed to $135^{\circ}$ rather than to $120^{\circ}$ to avoid a posterior blowout and to create the tunnel outlet under the soft tissues. In contrast to these reports, the present study showed that AM femoral drilling was safer at $120^{\circ}$ flexion than at $135^{\circ}$ flexion when avoiding femoral tunnel collision between ACL and ALL tunnels. Therefore, further studies are needed to investigate the safest femoral drilling procedure during combined double-bundle ACL and ALL reconstruction to prevent femoral tunnel collisions, including all of the lateral anatomic structure injuries.

Moreover, when it is difficult to achieve $135^{\circ}$ flexion (for instance owing to joint contracture, thick legs, or a hanging leg position with leg holder), the outlet points of PL drilling should be oriented more proximally to the ALL footprint, resulting in a higher risk of femoral tunnel collision between ACL and ALL tunnels. Although the outside-in technique, which allows the point of the outlets to be controlled on the femoral lateral surface, may be considered, this technique leads to acute graft bending, which is associated with poor graft maturation. ${ }^{35}$ Therefore, for such knees, femoral drilling using the flexible reamer and curved guide system, which allows a certain degree of control over intra-articular tunnel orientation ${ }^{36}$ and can produce significantly more anteverted femoral tunnels than those obtained with a rigid drill $^{37}$ or other fixation methods such as anchors or staples, may be better.

This study had some limitations. First, we had a small sample size. Second, we excised structures surrounding the knee (e.g., the skin, patella, and quadriceps tendon) that may have had an influence on the knee joint alignment during femoral drilling. Third, although simulations for the tunnels utilized for reconstruction grafts were performed by drawing the diameters of tunnels and outlets as circles, these might actually be ellipses (i.e., imperfect circles), because most ACL and ALL drilling is not perpendicular to the lateral surface of the femur or each other. Therefore, the values in this study might have been influenced by these slight differences from clinical operations. Fourth, the cadaver height and the size of the femoral condyle were not evaluated. These characteristics might have been associated with the chance of presenting collisions; thus, they may have affected our results. Finally, the original ALL in each knee was not identified because cadaveric knees embalmed with formaldehyde were used in this study; hence, the ALL footprint was determined as the position of the lateral epicondyle. Future studies using fresh frozen cadaveric knees are required to identify the original ALL. 27,28

In conclusion, with a narrow safe zone during only PL135/ AM120 combination, the risk of femoral tunnel collisions in combined double-bundle ACL and ALL reconstruction is high. AM drilling at $120^{\circ}$ and PL drilling at $>135^{\circ}$ knee flexion, combined with ALL drilling at $30^{\circ}$ coronal angle and $>45^{\circ}$ axial angle, may reduce this risk. These findings may enable the combined double-bundle ACL and ALL reconstruction to be safely and rapidly performed by reducing surgeons' concerns regarding femoral tunnel collision.

\section{Ethical Approval}

All procedures were performed at the dissection room of the Nagoya City University, and ethical approval was obtained (IRB number 60-18-0140).

\section{Funding \\ None.}

\section{Conflict of Interest \\ None declared.}

\section{Acknowledgments}

The authors would like to express their special thanks toT.U. (Department of Integrative Anatomy) and S.U.(Department of Anatomy and Neuroscience) for their support of the gross anatomy course at our facility.

\section{References}

1 Ardern CL, Webster KE, Taylor NF, Feller JA. Return to sport following anterior cruciate ligament reconstruction surgery: a systematic review and meta-analysis of the state of play. $\mathrm{Br} \mathrm{J}$ Sports Med 2011;45(07):596-606

2 Rasmussen MT, Nitri M, Williams BT, et al. An in vitro robotic assessment of the anterolateral ligament, part 1: secondary role of the anterolateral ligament in the setting of an anterior cruciate ligament injury. Am J Sports Med 2016;44(03):585-592

3 Tavlo M, Eljaja S, Jensen JT, Siersma VD, Krogsgaard MR. The role of the anterolateral ligament in ACL insufficient and reconstructed knees on rotatory stability: A biomechanical study on human cadavers. Scand J Med Sci Sports 2016;26(08):960-966

4 Thein R, Boorman-Padgett J, Stone K, Wickiewicz TL, Imhauser $\mathrm{CW}$, Pearle AD. biomechanical assessment of the anterolateral ligament of the knee: a secondary restraint in simulated tests of the pivot shift and of anterior stability. J Bone Joint Surg Am 2016; 98(11):937-943

5 Sobrado MF, Giglio PN, Bonadio MB, et al. Outcomes after isolated acute anterior cruciate ligament reconstruction are inferior in patients with an associated anterolateral ligament injury. Am J Sports Med 2020;48(13):3177-3182

6 Nitri M, Rasmussen MT, Williams BT, et al. An in vitro robotic assessment of the anterolateral ligament, part 2: anterolateral ligament reconstruction combined with anterior cruciate ligament reconstruction. Am J Sports Med 2016;44(03):593-601

7 Chen J, Xu C, Cho E, Huangfu X, Zhao J. Reconstruction for chronic ACL tears with or without anterolateral structure augmentation 
in patients at high risk for clinical failure: a randomized clinical trial. J Bone Joint Surg Am 2021;103(16):1482-1490

8 Saithna A, Daggett M, Helito CP, et al. Clinical results of combined $\mathrm{ACL}$ and anterolateral ligament reconstruction: a narrative review from the SANTI study group. J Knee Surg 2021;34(09):962-970

9 Sonnery-Cottet B, Saithna A, Cavalier M, et al. anterolateral ligament reconstruction is associated with significantly reduced ACL graft rupture rates at a minimum follow-up of 2 years: a prospective comparative study of 502 patients from the SANTI study group. Am J Sports Med 2017;45(07):1547-1557

10 Helito CP, Sobrado MF, Giglio PN, et al. Combined reconstruction of the anterolateral ligament in patients with anterior cruciate ligament injury and ligamentous hyperlaxity leads to better clinical stability and a lower failure rate than isolated anterior cruciate ligament reconstruction. Arthroscopy 2019;35(09): 2648-2654

11 Chahla J, Menge TJ, Mitchell JJ, Dean CS, LaPrade RF. Anterolateral ligament reconstruction technique: an anatomic-based approach. Arthrosc Tech 2016;5(03):e453-e457

12 Smith JO, Yasen SK, Lord B, Wilson AJ. Combined anterolateral ligament and anatomic anterior cruciate ligament reconstruction of the knee. Knee Surg Sports Traumatol Arthrosc 2015;23(11): 3151-3156

13 Wagih AM, Elguindy AM. Percutaneous reconstruction of the anterolateral ligament of the knee with a polyester tape. Arthrosc Tech 2016;5(04):e691-e697

14 Smeets K, Bellemans J, Lamers G, et al. High risk of tunnel convergence during combined anterior cruciate ligament and anterolateral ligament reconstruction. Knee Surg Sports Traumatol Arthrosc 2019;27(02):611-617

15 Gali JC, Bernardes AdeP, dos Santos LC, Ferreira TC, Almagro MA, da Silva PA. Tunnel collision during simultaneous anterior cruciate ligament and posterolateral corner reconstruction. Knee Surg Sports Traumatol Arthrosc 2016;24(01):195-200

16 Gelber PE, Erquicia JI, Sosa G, et al. Femoral tunnel drilling angles for the posterolateral corner in multiligamentary knee reconstructions: computed tomography evaluation in a cadaveric model. Arthroscopy 2013;29(02):257-265

17 Osaki K, Okazaki K, Tashiro Y, Matsubara H, Iwamoto Y. Influences of knee flexion angle and portal position on the location of femoral tunnel outlet in anterior cruciate ligament reconstruction with anteromedial portal technique. Knee Surg Sports Traumatol Arthrosc 2015;23(03):777-784

18 Jette C, Pomés J, Sastre S, Gutierrez D, Llusa M, Combalia A. Safe drilling angles avoid femoral tunnel complications during combined anterolateral ligament and anterior cruciate ligament reconstruction. Knee Surg Sports Traumatol Arthrosc 2019;27 (11):3411-3417

19 Järvelä S, Kiekara T, Suomalainen P, Järvelä T Double-bundle versus single-bundle anterior cruciate ligament reconstruction: a prospective randomized study with 10 -year results. Am J Sports Med 2017;45(11):2578-2585

20 El-Sherief FAH, Aldahshan WA, Wahd YE, et al. Double-bundle anterior cruciate ligament reconstruction is better than singlebundle reconstruction in terms of objective assessment but not in terms of subjective score. Knee Surg Sports Traumatol Arthrosc 2018;26(08):2395-2400

21 Maeyama A, Hoshino Y, Kato Y, et al. Anatomic double bundle ACL reconstruction outperforms any types of single bundle ACL reconstructions in controlling dynamic rotational laxity. Knee Surg Sports Traumatol Arthrosc 2018;26(05):1414-1419
22 Piefer JW, Pflugner TR, Hwang MD, Lubowitz JH. Anterior cruciate ligament femoral footprint anatomy: systematic review of the 21st century literature. Arthroscopy 2012;28(06):872-881

23 Nakamura M, Deie M, Shibuya $\mathrm{H}$, et al. Potential risks of femoral tunnel drilling through the far anteromedial portal: a cadaveric study. Arthroscopy 2009;25(05):481-487

24 Otani M, Nozaki M, Kobayashi M, et al. Comparative risk of common peroneal nerve injury in far anteromedial portal drilling and transtibial drilling in anatomical double-bundle ACL reconstruction. Knee Surg Sports Traumatol Arthrosc 2012;20(05): 838-843

25 Basdekis G, Abisafi C, Christel P. Effect of knee flexion angle on length and orientation of posterolateral femoral tunnel drilled through anteromedial portal during anatomic double-bundle anterior cruciate ligament reconstruction. Arthroscopy 2009;25 (10):1108-1114

26 Farrow LD, Parker RD. The relationship of lateral anatomic structures to exiting guide pins during femoral tunnel preparation utilizing an accessory medial portal. Knee Surg Sports Traumatol Arthrosc 2010;18(06):747-753

27 Kennedy MI, Claes S, Fuso FA, et al. The anterolateral ligament: an anatomic, radiographic, and biomechanical analysis. Am J Sports Med 2015;43(07):1606-1615

28 LaPrade RF, Ly TV, Wentorf FA, Engebretsen L. The posterolateral attachments of the knee: a qualitative and quantitative morphologic analysis of the fibular collateral ligament, popliteus tendon, popliteofibular ligament, and lateral gastrocnemius tendon. Am J Sports Med 2003;31(06):854-860

29 Kanda Y. Investigation of the freely available easy-to-use software 'EZR' for medical statistics. Bone Marrow Transplant 2013;48(03): 452-458

30 Jaecker V, Ibe P, Endler CH, Pfeiffer TR, Herbort M, Shafizadeh S. High risk of tunnel convergence in combined anterior cruciate ligament reconstruction and lateral extra-articular tenodesis. Am J Sports Med 2019;47(09):2110-2115

31 Angelini FJ, Helito CP, Tozi MR, et al. Combined reconstruction of the anterior cruciate ligament and posterolateral corner with a single femoral tunnel. Arthrosc Tech 2013;2(03):e285-e288

32 Bonadio MB, Helito CP, Foni NO, et al. Combined reconstruction of the posterior cruciate ligament and medial collateral ligament using a single femoral tunnel. Knee Surg Sports Traumatol Arthrosc 2017;25(10):3024-3030

33 Sonnery-Cottet B, Daggett M, Helito CP, Fayard JM, Thaunat M. Combined anterior cruciate ligament and anterolateral ligament reconstruction. Arthrosc Tech 2016;5(06):e1253-e1259

34 Mediavilla I, Aramberri M, Tiso G, Murillo-González JA. Combined double bundle anterior cruciate ligament reconstruction and anterolateral ligament reconstruction. Arthrosc Tech 2018;7 (08):e881-e886

35 Ahn JH, Jeong HJ, Lee YS, Park JH, Lee JH, KoTS. Graft bending angle is correlated with femoral intraosseous graft signal intensity in anterior cruciate ligament reconstruction using the outside-in technique. Knee 2016;23(04):666-673

36 Wang JH, Lee DK, Chung ST, Lee BH. Influence of change of tunnel axis angle on tunnel length during double-bundle ACL reconstruction via the transportal technique. BMC Musculoskelet Disord 2017;18(01):237

37 Wein F, Osemont B, Goetzmann T, et al. Anteversion and length of the femoral tunnel in ACL reconstruction: in-vivo comparison between rigid and flexible instrumentation. J Exp Orthop 2019;6 (01):26 